\title{
Estudio de variables relevantes en el uso de la información publicada por los organismos públicos en Internet. Una reivindicación de la mediación periodística
}

\section{Study of relevant variables in the use of information published by public agencies on the Internet. $A$ claim of the role of journalists as social mediators}

\author{
Jose A. Ruiz San Roman \\ Profesor Titular Departamento de Sociología VI \\ (Universidad Complutense de Madrid) \\ Gaspar Brändle \\ Profesor Contratado Doctor Departamento de Sociología \\ (Universidad de Murcia) \\ María Dolores Cáceres \\ Profesora Titular Departamento de Sociología IV \\ (Universidad Complutense de Madrid)
}

Fecha de recepción: 27 de enero de 2016

Fecha de revisión: 25 de junio de 2017

Fecha de publicación: 1 de julio de 2017

Para citar este artículo: Ruiz San Roman, J. A., Brändle, G. y Cáceres, M. D. (2017):

Estudio de variables relevantes en el uso de la información publicada por los organismos públicos en Internet. Una reivindicación de la mediación periodística, Icono 14, volumen 15 (2), pp. 137-157. doi: 10.7195/ri14.v15i2.1068 


\section{ARTÍCULOS DE INVESTIGACIÓN}

\section{Resumen}

Las políticas de transparencia han hecho que muchas instituciones públicas ofrezcan en Internet información abundante y relevante. Este texto estudia el uso que hace la ciudadanía de esa información. La metodología parte de una encuesta a usuarios españoles, haciendo un análisis mediante regresión logística con el objetivo de establecer cuáles son las variables que más determinan el uso ciudadano de la información difundida por organismos públicos en Internet. El modelo obtenido evidencia la relevancia de seis factores: alfabetización digital especializada, sexo, participación en asuntos públicos a través de Internet, nivel de estudios, edad y percepción de las posibilidades que ofrecen las instituciones públicas para acceder a sus datos. Al ser la alfabetización digital especializada el factor más relevante, se concluye en la necesidad de fomentar tanto la alfabetización de la ciudadanía en general como la de los periodistas en particular, dada su importancia como agentes mediadores especializados en este ámbito.

Palabras clave: Periodismo - Alfabetización - Digital - Ciudadanía - Transparencia

\section{Abstract}

The transparency policies have made many public institutions offer abundant and relevant information on the Internet. This paper studies the use of this information by citizens. The methodology is based on a survey of Internet users in Spain, analyzing the data through logistic regression in order to establish the variables that most determine the citizen use of the information disseminated by public institutions on the Internet. Six factors are relevant in the model: Specialized digital literacy, Sex, Participation in public affairs through the Internet, Level of education, Age and Perception of the possibilities offered by public institutions to access their data. Specialized digital literacy is the most relevant factor. Accordingly, this research asks to promote specialized literacy of citizens, and particularly journalists, because of their importance as specialized mediators.

Key Words: Journalism - Digital - Literacy - Citizenship - Transparency 


\section{Introducción. La transparencia como impulso de la publicación de información por parte de los organismos públicos en Internet}

Los organismos públicos disponen de mucha información para el desempeño eficaz de las actividades que tienen encomendadas. Basta pensar en la gran cantidad de información que recoge, usa y almacena un juzgado, una empresa municipal de transporte, los servicios meteorológicos, el sistema educativo o el parlamento, por señalar servicios públicos muy diversos. Con acierto, se ha subrayado la necesidad de que se desarrollen políticas de transparencia de modo que la información de las instituciones públicas esté disponible para el uso por parte de toda la ciudadanía. El argumento central es sencillo: la información de los organismos públicos es de todos $\mathrm{y}$, en consecuencia, se debe permitir a todos su uso (Ley de transparencia, acceso a la información pública y buen gobierno, 2013). Ciertamente, tanto la acumulación, como la puesta en circulación de datos ocasiona problemas diversos que van desde la protección de la intimidad, a la sostenibilidad económica o a los problemas de seguridad de las transferencias internacionales, etc. (Zwolinska, 2015). De modo que el principio general que legitima el uso de las informaciones y datos de los organismos públicos puede tener algunas excepciones cuando expresamente se justifique, pero deberán ser consideradas estrictamente como excepciones, que no alteran sustancialmente el principio general.

La transparencia de los organismos públicos tiene consecuencias políticas y comunicativas significativas. Una de las más destacadas es que permite un mejor control de las actividades del poder. Es lógico que se consideren las iniciativas de transparencia entre las medidas relevantes encaminadas a la mejora de la calidad democrática y a la lucha contra la corrupción (Bertot, Jaeger, \& Grimes, 2010) en coherencia con el marco teórico de la "democracia monitorizada". Entendiendo por monitorización "el ejercicio de escrutinio público hacia los centros y las relaciones de poder" (Keane \& Feenstra, 2014, pág. 49).

La importancia de la transparencia de las organizaciones públicas para la salud democrática hace que las administraciones públicas deban comprometerse en la promoción de la publicación de la información lo que supone no sólo permitir el 


\section{ARTÍCULOS DE INVESTIGACIÓN}

uso, sino que se debe promover positivamente la utilización de la información disponible (Gértrudix, Gertrudis-Casado \& Álvarez-García, 2016).

Las voces que defienden la necesidad de que la información que poseen las instituciones públicas sea accesible a toda la ciudadanía reciben un notable impulso a partir de la Ley 19/2013, de transparencia, acceso a la información pública y buen gobierno que señala que

la transparencia, el acceso a la información pública y las normas de buen gobierno deben ser los ejes fundamentales de toda acción política. Sólo cuando la acción de los responsables públicos se somete a escrutinio, cuando los ciudadanos pueden conocer cómo se toman las decisiones que les afectan, cómo se manejan los fondos públicos o bajo qué criterios actúan nuestras instituciones podremos hablar del inicio de un proceso en el que los poderes públicos comienzan a responder a una sociedad que es crítica, exigente y que demanda participación de los poderes públicos (Ley 19/2013, preámbulo).

Las instituciones públicas han creado portales para difundir información propia (y ajena) y promover su uso. En el ámbito europeo destaca, por ejemplo, el Portal de Datos Abiertos de la Unión Europea. Portales semejantes se pueden encontrar en ámbitos locales o nacionales (Gobierno de España). En los portales de transparencia se publica información sobre su uso: número de páginas vistas, evolución del número de usuarios, etc. Sirva como ejemplo lo publicado en http://transparencia.gob.es/transparencia/transparencia_Home/index/MasSobreTransparencia/ El-portal-en-cifras.html

Desde el punto de vista de los ciudadanos resulta razonable pensar que se tomarán mejores decisiones (al menos, decisiones más informadas) si es posible utilizar información de calidad sobre la realidad social. En esa línea, iniciativas sociales o movimientos como el denominado "movimiento open data" abogan por la necesidad de poner a disposición de la ciudadanía cuantos más datos mejor (Garrigá-Portolà, 2011).

Las investigaciones empíricas corroboran las posibilidades de la ciudadanía de participar activamente en las decisiones que afectan a todos. Baste como ejemplo 
la investigación realizada entre los jóvenes españoles en la que declaran "utilizar las redes sociales para apoyar campañas de solidaridad (34,3\%), para denunciar situaciones injustas $(27,2 \%)$ o los que aseguran que las redes sociales los han llevado a participar en alguna protesta social (27\%) (García-Galera, Del-Hoyo-Hurtado, \& Fernández-Muñoz, 2014).

De ahí que se insista en que no basta con la "puesta a disposición" de la información y que la use quien sepa y quiera, sino que es preciso que, una vez que los datos han sido publicados y están disponibles para cualquiera que quiera utilizarlos, hay que preguntarse si esa información está siendo realmente utilizada. No se puede esquivar la cuestión de si resulta útil publicar información disponible en las instituciones públicas o, por el contrario, está siendo, de hecho, inútil porque son pocos los que tienen algún interés o mínima capacidad para usarla.

Podría ocurrir que la fiscalización de la labor pública mediante la utilización de información cada vez más accesible sea teóricamente un importante avance. Pero si únicamente una pequeña parte de la población se interesa por este tipo de información publicada por los organismos públicos, dicha publicación podría llegar a convertirse en un asunto irrelevante o, aún peor, que actuara como un narcótico que aplaca las capacidades de participación social (efecto narcotización) (Arroyo, 2014). Podría ser un modo de sensibilidad hacia las demandas participativas de la ciudadanía, pero sin consecuencias reales para el control social del poder político.

De hecho, cuando en esta investigación se ha preguntado a los encuestados sobre el uso de datos en abierto (salarios, presupuestos, etc.) publicados por organismos públicos se obtuvieron los siguientes resultados: el 20,6\% sí ha utilizado datos abiertos publicados por instituciones públicas, el $41,2 \%$ no ha utilizado datos abiertos publicados por instituciones públicas pero sí los conocen y el 38,2\% ni siquiera conoce los datos abiertos publicados por instituciones públicas (Cáceres, Brändle, \& Ruiz San Román, 2017).

En consecuencia, desde estos presupuestos, en este texto se analizan las variables que pueden explicar el uso (o, en su caso el "no uso") que hacen los ciudadanos de la información puesta a disposición por los organismos públicos. Y, a partir 


\section{ARTÍCULOS DE INVESTIGACIÓN}

de ahí, se detecta la relevancia de la alfabetización especializada, lo que supone reivindicar la figura del profesional de la información como actor clave en el proceso de empoderamiento ciudadano en el marco de la profunda crisis/transformación del entorno socio-comunicativo (Thompson, 2008).

\section{Objetivo e hipótesis}

El objetivo de este análisis es mostrar las variables que mejor colaboran a explicar el uso (o no uso) que hacen los ciudadanos de la información (salarios, contratos, estadísticas, presupuestos, etc.) que los organismos públicos ponen a su disposición a través de Internet.

La hipótesis principal que se trata de contrastar, de acuerdo con el objetivo explicitado, es que a la hora de explicar el uso de información publicada online por los organismos públicos existen distintas variables de influencia, tanto personales como contextuales, que determinarían el uso o no uso de esos datos.

\section{Método}

Es preciso aclarar que este texto se refiere al conjunto de informaciones publicadas por los organismos públicos y no sólo a lo que técnicamente se denomina "open data", es decir, a datos disponibles en bruto que pueden ser leídos y reutilizados automáticamente por máquinas (Ferrer-Sapena, Peset, \& Aleixandre-Benavent, 2011). Ciertamente en algunas de las preguntas utilizadas en el cuestionario sobre el que se sustenta esta investigación, se utiliza el término "datos en abierto" que podría interpretarse como equivalente a "open data". Sin embargo, si se atiende a la pregunta completa en su estricta literalidad, lo realmente preguntado se ajusta no tanto a lo que técnicamente se llama "open data" sino más bien a lo que en este artículo se denomina "información publicada por los organismos públicos en Internet".

Un ejemplo puede resultar útil para ilustrar esta aclaración conceptual. La pregunta del cuestionario que se usa como variable dependiente dice literalmente " ¿Ha accedido o utilizado alguna vez datos en abierto (salarios, contratos, 
estadísticas, presupuestos...) publicados por organismos públicos españoles, europeos o internacionales?". Como se puede ver se utiliza el término "datos en abierto" unido a una detallada explicación "salarios, contratos, estadísticas, presupuestos... publicados por organismos públicos españoles, europeos 0 internacionales" con lo que se deja claramente abierta la posibilidad a contestar afirmativamente si alguien ha consultado datos sobre salarios de ministros, evolución del desempleo, disposiciones legales, acuerdos del ayuntamiento, etc. que, obviamente, no tienen que tener un formato de base de datos reutilizable por máquinas, por ejemplo. Es decir, no tienen por qué ajustarse al concepto generalmente aceptado de "open data".

La investigación está basada en una encuesta propia realizada en España a usuarios de Internet, de ambos sexos, mayores de 16 años. Se realizaron entrevistas telefónicas a partir de un cuestionario estructurado que cuenta con 33 preguntas, a una muestra de la población española de 1183 sujetos. El trabajo de campo se realizó del 6 al 17 de octubre de 2014. El error muestral, para un nivel de confianza del $95,5 \%$ (dos sigmas), y $\mathrm{P}=0$, es de $\pm 3,16 \%$ para el conjunto de la muestra y en el supuesto de muestreo aleatorio simple. Si bien el cuestionario utilizado contaba con 33 preguntas, para este trabajo se han identificado aquellas variables que, de manera teórica, mejor se ajustaran al problema y los objetivos planteados. En el anexo de este trabajo puede consultarse un resumen descriptivo de las variables utilizadas en esta investigación.

\section{Variable dependiente}

Acceso y uso de datos en abierto de organismos públicos a través de Internet. Atendiendo al objetivo planteado, se incluyó en el cuestionario una pregunta que trataba de conocer el grado de acceso y uso de los datos en abierto (salarios, presupuestos, etc.) que ofrecen los organismos públicos nacionales e internacionales. La pregunta que inicialmente tenía tres categorías de respuesta: "Sí, he accedido y los he utilizado", "Los conozco, pero no los he utilizado", "No los conozco", fue tratada para convertirla en una variable ficticia o dummy ( 0 = No uso; 1 = Sí uso) que permitiera ser utilizada en una regresión logística como variable dependiente (Tabla 2). 


\section{ARTÍCULOS DE INVESTIGACIÓN}

\section{Variables independientes}

Participación en asuntos públicos a través de Internet. Se creó un índice compuesto con todas aquellas variables que medían el nivel de participación de los ciudadanos en actividades públicas a través de Internet. Esta nueva variable se formó con 8 ítems que evaluaban el grado de participación de los encuestados en una escala Likert de 5 puntos ( $1=$ nunca, a $5=$ muchas veces $)$. La consistencia de la nueva medida compuesta es elevada $(\alpha=.90)$ y por ello se procedió a utilizarla como una nueva variable.

Expectativas de repercusión de las acciones realizadas a través de Internet. Con el objetivo de medir el nivel de expectativas que tienen los ciudadanos hacia la repercusión que pueden tener las acciones públicas realizadas a través de Internet se creó un índice combinado. Esta nueva variable se formó con 8 ítems que evaluaban el grado de expectativas de los encuestados en una escala Likert de 5 puntos ( 1 = ninguna repercusión, a $5=$ mucha repercusión). Los ítems mostraron una alta consistencia interna $(\alpha=.89)$.

Competencia digital básica. Siguiendo el mismo procedimiento se creó una variable compuesta por aquellos ítems que medían el grado de competencia que reconocían los encuestados en el uso de herramientas digitales básicas. Esta nueva variable es resultado de la combinación de 5 ítems que medían el nivel de competencia digital en una escala Likert de 5 puntos $(1=$ nada, a $5=$ mucho $)$. La alta intercorrelación entre las variables $(\alpha=.92)$ indica que es oportuno tomarlas como una nueva medida compuesta.

Visibilidad y posibilidad de acceso a los datos abiertos de instituciones públicas. A fin de medir la percepción que tienen los ciudadanos sobre el grado en el que las instituciones públicas están visibles en Internet y ofrecen datos de carácter abierto sobre su funcionamiento se creó una medida compuesta por 4 ítems que evaluaban dichos aspectos en una escala Likert de 5 puntos ( $1=$ totalmente en desacuerdo, a $5=$ totalmente de acuerdo). La fiabilidad de la nueva medida compuesta es elevada $(\alpha=.89)$ y por ello se procedió a utilizarla como una nueva variable.

Alfabetización para el uso de información pública en internet. Con el objetivo de estimar el nivel de alfabetización que los encuestados consideran tener en la utilización de la información pública que ofrecen las instituciones en Internet, se construyó una nueva medida compuesta por 8 ítems que medían mediante escala Likert de 5 puntos 
( 1 = nada preparado, a $5=$ muy preparado). La alta consistencia interna de la nueva variable $(\alpha=.93)$ hace pertinente su utilización.

Sexo. Variable nominal con dos valores: Hombre y Mujer.

Edad. Compuesta por seis categorías: entre 16 y 18 años, entre 19 y 24 años, entre 25 y 34 años, entre 35 y 44 años, entre 45 y 54 años, más de 55 años.

Nivel de estudios. La variable tiene las siguientes categorías: Sin estudios, Primaria, Secundaria obligatoria, Formación Profesional de Grado Medio, Bachillerato, Formación Profesional de Grado Superior, y Universitarios.

\section{Análisis y resultados}

Este estudio da cuenta de las variables que mejor explican el acceso y uso de los datos en abierto que ofrecen los organismos públicos, es decir, trata de determinar en qué circunstancias los ciudadanos utilizarán (o no) estos datos (salarios, contratos, presupuestos, etc.). Se ha realizado un análisis de regresión logística, que permite estudiar la relación entre variables aportando información sobre en qué medida la variable dependiente (categórica dicotómica) puede estar explicada por la(s) variable(s) independientes (Brändle, 2011).

Se toma como variable dependiente el acceso y uso de datos en abierto de organismos públicos a través de Internet, y como variables predictoras o independientes (Tabla 3 y 4), todas aquellas variables personales y contextuales medidas en la investigación y que, a priori, podrían considerarse como influyentes en la explicación del nivel de participación. El análisis realizado posibilita comprobar la interacción de variables de tipo endógeno o individual, como las características sociodemográficas de la persona, con variables exógenas o contextuales, como las posibilidades que ofrecen las instituciones públicas para acceder a sus datos. Por último, y si bien el modelo final no es perfecto al no ser posible determinar de manera unívoca y definitiva todas las variables que influyen en el uso que los ciudadanos hacen de los datos en abierto, sí se consigue establecer la importancia (o irrelevancia) explicativa de una serie de las variables analizadas. La tabla 1 muestra el modelo de regresión 
logística. Podemos distinguir tres columnas: la primera presenta el conjunto de variables independientes que forman el modelo; la segunda presenta los coeficientes $B$ (logits), que son los coeficientes que dan origen a la ecuación de regresión; la tercera presenta los mismos coeficientes B, en este caso estandarizados, lo que permite interpretar de forma más directa la variación experimentada por la variable dependiente, en función de los cambios producidos en las distintas variables independientes.

\begin{tabular}{|c|c|c|}
\hline Variables & Coeficiente B & $\operatorname{Exp}(B)$ \\
\hline Alfabetización para el uso de información pública en internet & $\begin{array}{c}0,999 \\
(0,180)^{\star *}\end{array}$ & 2,716 \\
\hline Expectativas de repercusión de las acciones a través de Internet & $\begin{array}{c}0,006 \\
(0,125)^{\mathrm{ns}}\end{array}$ & 1,006 \\
\hline Participación en asuntos públicos a través de Internet & $\begin{array}{c}0,332 \\
(0,113)^{\star *}\end{array}$ & 1,394 \\
\hline $\begin{array}{l}\text { Visibilidad y posibilidad de acceso a los datos abiertos de } \\
\text { instituciones públicas }\end{array}$ & $\begin{array}{c}0,255 \\
(0,093)^{* *}\end{array}$ & 1,291 \\
\hline Competencia digital básica & $\begin{array}{c}-0,170 \\
(0,165)^{\text {ns }}\end{array}$ & 0,844 \\
\hline Sexo & $\begin{array}{c}0,427 \\
(0,174)^{*}\end{array}$ & 1,533 \\
\hline Edad & $\begin{array}{l}-0,174 \\
(0,06)^{\star *}\end{array}$ & 0,840 \\
\hline Nivel de estudios & $\begin{array}{c}0,242 \\
(0,071)^{\star *} \\
\end{array}$ & 1,274 \\
\hline Constante & $\begin{array}{c}-6,848 \\
(0,827)^{\star *}\end{array}$ & ,001 \\
\hline
\end{tabular}

\begin{tabular}{|r|c|}
\hline $\mathrm{N}$ & 927 \\
\hline Porcentaje de casos predichos & $78,7 \%$ \\
\hline Seudo r-cuadrado (Nagelkerke) & 0,210 \\
\hline
\end{tabular}

Tabla 1: Regresión logística. Variables explicativas del uso de los datos e información en abierto que ofrecen los organismos públicos a través de Internet Variable dependiente: Acceso y uso de datos en abierto de organismos públicos a través de Internet Errores típicos entre paréntesis: ${ }^{* *}(p<0,01) ;{ }^{*}(p<0,05) ; n s \quad(p>0,05)$

Fuente: elaboración propia. 
Para evaluar la intensidad de la relación entre dos variables del modelo podemos fijarnos en la columna $\operatorname{Exp}(\mathrm{B})$. Así, por ejemplo, elevar en un punto el nivel de alfabetización para el uso de información pública en Internet multiplica por 2,716 la probabilidad de una persona de acceder y utilizar datos en abierto de organismos públicos. En el caso de valores por debajo de 1, éstos indican una reducción en la variable dependiente por unidad de variación en la variable independiente.

Atendiendo al comportamiento de cada una de las variables independientes e interpretando la intensidad y la significatividad de los coeficientes de regresión, se puede afirmar que las variables que influyen en el acceso y uso de los datos en abierto que ofrecen los organismos públicos a través de Internet son, por orden de intensidad: la Alfabetización para el uso de información pública en internet, el Sexo del encuestado, la Participación en asuntos públicos a través de Internet, la Visibilidad y posibilidad de acceso a los datos abiertos de instituciones públicas, el Nivel de estudios y la Edad. Mientras que quedarían fuera del modelo, no colaborando a la explicación de la variable dependiente, las Expectativas de repercusión de las acciones a través de Internet y la Competencia digital básica.

\section{Discusión}

El análisis expuesto permite mostrar que hay seis variables que tienen una influencia significativa para explicar el uso ciudadano de la información publicada en Internet por los organismos públicos.

Comenzando por aquella que más colabora a la construcción del modelo, se podría decir que el nivel de alfabetización en el uso y gestión de la información pública (alfabetización digital especializada) tiene una influencia decisiva. Estos resultados están en la línea de la literatura científica sobre la materia, que sostiene la necesidad de alfabetización digital para el desarrollo de habilidades tecnológicas de cierta complejidad (Pérez Tornero, 2015; Ferrés, Aguaded, \& García Matilla, 2012). Una conclusión inmediata podría ser la necesidad de impulsar iniciativas de alfabetización digital especializada para el conjunto de la ciudadanía. Sin embargo, conviene tener presente el notable desinterés declarado por los jóvenes hacia las acciones de alfabetización digital sobre datos abiertos (Gértrudix, Gálvez 


\section{ARTÍCULOS DE INVESTIGACIÓN}

de la Cuesta, Said, \& Durán Medina, 2016). Esto obliga a plantear estrategias de alfabetización que tengan en cuenta la desidia del público general y se centren en actores especializados. Más adelante se detalla la propuesta de alfabetización de los profesionales de la información como mediadores cualificados.

Con una influencia explicativa algo menor estaría la variable sexo. Los hombres acceden y usan más la información de los organismos públicos publicada en Internet. Estos resultados evidencian la permanencia de la brecha digital de género y la necesidad de políticas públicas correctoras (Gil-Juárez, Vitores, \& Feliu, 2012).

Le seguiría en nivel de capacidad explicativa la variable participación en asuntos públicos a través de Internet, de forma que las personas que más participan a través de la Red, serían también aquellas que utilizan más la información en abierto que ofrecen los organismos públicos.

Con una aportación similar al modelo, estarían las posibilidades que ofrecen las instituciones públicas para conocer y acceder a sus datos. Esto avala la importancia de las políticas encaminadas a que los usuarios perciban que la información de las instituciones públicas sea accesible (OECD, 2008).

El nivel de estudios también tendría una aportación significativa a la hora de explicar la variable dependiente, de manera que un mayor nivel de estudios alcanzado supone más utilización de la información puesta a disposición por las instituciones públicas.

Por último, y con una capacidad explicativa algo menor, estaría la edad. Al tener un signo negativo, la interacción nos indica que entre los de menor edad, se da una mayor probabilidad de acceso y uso de la información ofrecida por las instituciones públicas. El dato resulta lógico y es coherente con los estudios que señalan la mayor facilidad de los nativos digitales para el manejo de cualquier herramienta tecnológica (Prenski, 2001; Ferrer y Ruiz, 2006; Gertrudix, 2009).

Sin embargo, dos de las variables que se consideró previamente que podrían ser significativas, una vez realizado el análisis, se observa que no colaboran a predecir 
el uso de información publicada por los organismos públicos: la competencia digital básica y las expectativas de repercusión de las acciones a través de Internet.

La explicación de la ausencia de influencia de la competencia digital básica en el modelo podría deberse a que dicha competencia, tal como se ha establecido, se ocupa de aspectos demasiado elementales. Prácticamente se limita a comprobar que el sujeto tiene unos rudimentos informáticos $\mathrm{y}$, por tanto, esta variable apenas discrimina dado que la población de la que se ha obtenido la muestra tiene esas competencias básicas al ser usuarios de Internet.

Sin embargo, resulta más difícil de explicar la cuestión de la irrelevancia de las expectativas de los usuarios sobre la influencia de sus acciones. Es posible que no se haya construido una variable adecuada para medir las expectativas de repercusión de las acciones en Internet y se deberían afinar los indicadores con los que se ha medido esta variable. Además, se debe tener en cuenta el entorno de notable desconfianza hacia las instituciones públicas a la hora de recoger los datos, llegándose a señalar a los responsables públicos como uno de los principales problemas del país (Centro de Investigaciones Sociológicas, 2015). Hasta que esa desconfianza en los responsables públicos se corrija habrá dificultades para trabajar con los datos de expectativas de influencia en las instituciones públicas.

El poder explicativo del modelo podría mejorarse con el rediseño de la variable sobre las expectativas de repercusión de las acciones en Internet y quizá, con la inclusión de otras variables tales como el interés en la participación, interés en la política o en las políticas sociales que podrán ser utilizadas en investigaciones posteriores similares.

\section{Relevancia para las políticas públicas de alfabetización digital. Reivindicación de los profesionales de la información}

Los resultados obtenidos resultan relevantes para la toma de decisiones sobre políticas públicas de transparencia de los organismos públicos, cuestión prioritaria desde la entrada en vigor de la Ley 19/2013 de transparencia y la consiguiente puesta en marcha del llamado "Portal de la Transparencia del Gobierno de España" (Gobierno de España). 
Como se ha señalado, las administraciones públicas deben centrar sus esfuerzos, no sólo en ofrecer cada vez más información para garantizar las exigencias sociales y legales de transparencia, sino también en capacitar personas para el uso de la información. Sin embargo, no debe obviarse el grado de complejidad de buena parte de la información publicada por los organismos públicos Así, no está al alcance del gran público manejar datos en bruto o determinados servicios digitales abiertos (García \& Gertrudix, 2011). Su uso no resulta espontáneo ni sencillo y, por consiguiente, como muestra nuestro análisis, no basta con tener conocimientos informáticos básicos, sino que su manejo requiere una alfabetización digital especializada. Ciertamente, la administración pública realiza esfuerzos interesantes por avanzar en este punto como los trabajos del "Proyecto Aporta" (http://datos.gob.es/es/documentacion).

La propuesta que se hace en este texto subraya la necesidad de que las políticas de alfabetización digital se marquen objetivos diversos en función de los públicos. Se trataría de plantear objetivos de alfabetización digital básica para la población general (nivel básico de sensibilización y conocimiento de la existencia y las posibilidades de la información disponible) y dedicar los esfuerzos de alfabetización especializada avanzada a los colectivos más capaces de realizar una función mediadora entre el público y los datos más complejos publicados. Entre esos colectivos están, incuestionablemente, los profesionales de la información. En todo proceso de apertura de información por parte de los organismos públicos los medios de comunicación tradicionales y alternativos y sus profesionales pueden y deben jugar un papel destacado (Kovach \& Roseenstiel, 2003).

Como es sabido, la publicación de notable cantidad de información y datos en bruto por organizaciones públicas y privadas está permitiendo la expansión del sector infomediario. También, en el mundo del Periodismo, el que empezó llamándose "periodismo de precisión" (Meyer, 1993; Dader García, 1997) y que en los últimos años ha dado en llamarse "periodismo de datos", resulta posible gracias a que periodistas con formación tecnológica suficiente utilizan el potencial de los datos abiertos y saben descubrir su interés informativo (Flores Vivar \& Salinas Aguilar, 2012).

En no pocas ocasiones, la información publicada por los organismos públicos tiene una notable complejidad técnica, de modo que la mediación no es un adorno prescindible. 
Cada vez más se trata de una mediación necesaria en los procesos de transparencia, como valor añadido de un servicio propio especializado de los profesionales de la información.

Sin embargo, la cuestión no puede darse por sabida porque la idea no parece haber sido asimilada por la comunidad científica. Sorprende que, en ocasiones, autores especialistas en la materia, al referirse a los "reutilizadores" de los datos abiertos, ni siquiera mencionen a los periodistas (Álvarez Espinar, 2014, págs. 19-20).

Los cambios que la innovación tecnológica ha impuesto a la profesión periodística están generando interesantes esfuerzos por repensar el sentido del quehacer periodístico (Rodriguez Brito \& García Chico, 2013). La Red ha traído consigo la crisis de las intermediaciones toda vez que permite, por una parte, que el receptor acceda a las fuentes sin necesidad de comunicadores profesionales $\mathrm{y}$, por otra, que los usuarios estén en posición de producir y distribuir contenidos al margen de los editores. Esto es, la Red permite que el ciudadano haga cosas que antes requerían la intervención de instituciones o procesos de intermediación, como informarse. Esto trae aparejados cambios en las disputas por el poder simbólico, habiéndose producido un desplazamiento desde el control sobre la información hacia la gestión de la información sobre la información. Pero, a la vez, y ésta es la interesante paradoja que se deriva de nuestro análisis, resulta patente la necesidad de mediadores especializados y críticos, con competencias técnicas y profesionales que actúen como intermediarios entre la ingente cantidad de información y una ciudadanía no siempre capaz de utilizarla, gestionarla y digerirla.

En consecuencia, la alfabetización digital especializada es una necesidad ineludible en la formación de los profesionales de la comunicación para trabajar con una creciente acumulación de datos e información procedente de los organismos públicos. En este momento de sobreabundancia de información, cobra especial relevancia el papel de los profesionales de la comunicación y el periodismo interpretativo que persigue, no solo dar a conocer sino, a través de la profundización, análisis y contextualización, ayudar a entender y dar sentido al mundo que nos rodea y así conseguir una ciudadanía con criterios que orienten la toma de decisiones y la acción sobre la realidad. 


\section{ARTÍCULOS DE INVESTIGACIÓN}

\section{Referencias}

Álvarez Espinar, M. (2014). Apertura y reutilización de datos públicos. En M. Álvarez Espinar, Govern Obert 2014. Barcelona: Generalitat de Catalunya.

Arroyo, L. (2014). ¿La ciberutopía era esto? Softactivismo, tirbalismo, nueva censura y trivialización del espacio público. En R. Cotarelo, \& J. Olmeda, La democracia del siglo XXI. Política, medios de comunicación, internet y redes sociales. Madrid: Centro de Estudios Políticos y Constitucionales.

Bertot, J. C., Jaeger, P., \& Grimes, J. (2010). Using ICTs to create a culture of transparency: E-government and social media as openess and anticorruption tools for societies. Governmet Information Quarterly (27), 264-271. doi:10.1016/j.giq.2010.03.001

Brändle, G. (2011). Regression and Regression Analisys. En G. Ritzer, \& J. Ryan, Concise Encyclopedia of Sociology. Oxford: Willey-Blackwell.

Cáceres, M., Brändle, G., \& Ruiz San Román, J.A. (2017). Nuevos modelos de participación y empoderamiento ciudadano en Internet. Revista de la Asociación Española de Investigación de la Comunicación, 4(7), 22-28. Recuperado de http://www.revistaeic.eu/index.php/raeic/article/view/100

Centro de Investigaciones Sociológicas. (2015). Barómetro. Estudio 3082. Madrid: CIS. Dader García, J. (1997). Periodismo de precisión: la vía socioinformática de descubrir noticias. Madrid: Síntesis.

Ferrer-Sapena, A., Peset, F., \& Aleixandre-Benavent, R. (2011). Acceso a los datos públicos y su reutilización: open data y open goverment. El Profesional de la Información, 20(3), 260-269. doi: 10.3145/epi.2011.may.03

Ferrer, M., \& Ruiz, J. (2005). Uso de videojuegos en niños de 7 a 12 años. Una aproximación mediante encuesta. Revista de Comunicación y Nuevas tecnologías, 4(1), 205-216. doi: 10.7195/ri14.v4i1.404

Ferrés, J., Aguaded, I., \& García Matilla, A. (2012). La competencia mediática de la ciudadanía española. Competencias y retos. Icono 14, 10(3), 23-42. doi:10.7195/ri14.v10i3.201

Flores Vivar, J. M., \& Salinas Aguilar, C. (2012). Sinergias en la construcción del Nuevo Periodismo derivadas del Data Journalism y el Transmedia Journalism. Recuperado de http://www.2ip.es/ 
García, F., \& Gertrudix, M. (2011). Naturalez y características de los servicios y contenidos digitales abiertos. CIC Cuadernos de Información y Comunicación (16), 125-138. doi:10.5209/rev_CIYC.2011.v16.7

García-Galera, M. C., Del-Hoyo-Hurtado, M., \& Fernández-Muñoz, C. (2014). Jóvenes comprometidos en la Red: el papel de las redes sociales en la participación social activa. Comunicar, XXII (43), 35-43. doi:10.3916/C43-2014-03

Garrigá-Portolà, M. (2011). ¿Datos abiertos? Sí, pero de forma sostenible. El profesional de la Información, 20(3), 298-303. doi: 10.3145/epi.2011.may.03 Gertrudix-Barrio, M. (2009) Nativos digitales. Presentación. Icono 14. Revista de Comunicación y Tecnologías Emergentes, 7(1), 1-6. doi:10.7195/ri14.v7i1.494 Gértrudix, M., Gálvez de la Cuesta, M., Said, E., \& Durán Medina, J. (2016). Alfabetización digital, competencias mediáticas y Open Data. Revista Latinoamericana de Tecnología Educativa, 15(2), 113-121. doi:10.17398/1695288X.15.2.113

Gértrudix, M., Gertrudis-Casado, M.C., \& Álvarez-García, S. (2016). Consumo de datos abiertos de las instituciones públcas por parte de los ciudadanos españoles. $E l$ profesional de la información, 25(4), 535-544. doi:10.3145/epi.2016.jul.03

Gil-Juárez, A., Vitores, A., \& Feliu, J. (2012). Género y TIC: en torno a la brecha digital de género. Athenea Digital, 3-9.

Gobierno de España. (s.f.). Portal de Transparencia del Gobierno de España. Recuperado de http://transparencia.gob.es/en/

Keane, J., \& Feenstra, R. A. (2014). Nuevas formas de participación política en el marco de la era digital. Telos. Revista de Pensamiento sobre Comunicación, Tecnología y Sociedad (98), 48-57.

Kovach, B., \& Roseenstiel, T. (2003). Los elementos del periodismo. Madrid: Aguilar. Ley 19/2013, de 9 de diciembre, de transparencia, acceso a la información pública y buen gobierno. Boletín Oficial del Estado, 10 de diciembre de 2013, núm. 295. pp. 97922-97952.. Recuperado de http://www.boe.es/boe/dias/2013/12/10/ pdfs/BOE-A-2013-12887.pdf

Meyer, P. (1993). Periodismo de precisión: nuevas fronteras para la investigación periodística. Barcelona: Bosch.

OECD. (2008). Recommedation of the Council for enhanced access and more effective use of public sector information. Recuperado de http://www.oecd.org/internet/ ieconomy/40826024.pdf 


\section{ARTÍCULOS DE INVESTIGACIÓN}

Pérez Tornero, J. M. (2015). La emergencia de la alfabetización mediática e informacional. Un nuevo paradigma para las políticas públicas y la investigación. Telos. Cuadernos de Comunicación e Innovación (100), 99-102.

Prensky, M (2001). Digital Natives, Digital Immigrants. On the Horizon, 9 (5), 1-6. Rodriguez Brito, A., \& García Chico, L. (2013). Big Data y periodismo en el coninente americano. Cinco casos de estudio. Telos. Cuadernos de Comunicación e Innovación (95), 57-67.

Thompson, J. (2008). Ideología y cultura moderna. La Habana: Pablo de la Torriente Brau.

Zwolinska, M. (2015). International transfers of personal data. Toward a global consensus on data protection standards. Derecom (19), 165-181.

\section{Agradecimientos}

Los datos que aquí se presentan forman parte del proyecto I+D+i “Ciudadanía Digital y 0pen Data Access: empoderamiento ciudadano a través de los medios sociales en el entorno digital" financiado por el Ministerio de Economía y Competitividad del Gobierno de España. (ref. CS02012-30756)

\section{Anexo}

\begin{tabular}{|l|c|c|}
\hline & N & Porcentaje \\
\hline Acceso y uso de datos en abierto de organismos públicos & \multicolumn{2}{|c|}{} \\
\hline Sí, he accedido y los he utilizado (1) & 232 & 20,6 \\
\hline Los conozco, pero no los he utilizado (2) & 464 & 41,2 \\
\hline No los conozco (3) & 430 & 38,2 \\
\hline Total (dummy) & 1126 & 100 \\
\hline Acceso y uso de datos en abierto de organismos públicos & & \\
\hline Sí, he accedido y los he utilizado (1) & 232 & 20,6 \\
\hline No los he utilizado (0) & 894 & 79,4 \\
\hline Total & 1126 & 100 \\
\hline
\end{tabular}

Tabla 2: Frecuencias de la variable dependiente original y dummy 
Estudio de variables relevantes en el uso de la información publicada... | 155

ARTÍCULOS DE INVESTIGACIÓN

\begin{tabular}{|c|c|c|c|c|c|}
\hline & $\mathrm{N}$ & Mínimo & Máximo & Media & $\begin{array}{l}\text { Desv. } \\
\text { típica }\end{array}$ \\
\hline Participación Acciones en Internet $(\alpha=.90)$ & 1062 & 1 & 5 & 3,10 & ,96 \\
\hline Expresar su opinión & 1102 & 1 & 5 & 3,47 & 1,18 \\
\hline Compartir opiniones de otras personas & 1104 & 1 & 5 & 3,54 & 1,18 \\
\hline Realizar una petición a las instituciones públicas & 1101 & 1 & 5 & 2,95 & 1,27 \\
\hline Presentar una queja a las instituciones públicas & 1099 & 1 & 5 & 3,03 & 1,21 \\
\hline Formular una protesta a las instituciones públicas & 1094 & 1 & 5 & 2,93 & 1,26 \\
\hline $\begin{array}{l}\text { Apoyar iniciativas solidarias mediante la firma } \\
\text { una iniciativa solidaria }\end{array}$ & 1094 & 1 & 5 & 3,59 & 1,25 \\
\hline Inscribirse en programas de voluntariado & 1091 & 1 & 5 & 2,66 & 1,34 \\
\hline $\begin{array}{l}\text { Apoyar económicamente causas solidarias me- } \\
\text { diante donaciones }\end{array}$ & 1095 & 1 & 5 & 2,79 & 1,33 \\
\hline Expectativas Acciones en Internet $(\alpha=.90)$ & 1024 & 1 & 5 & 3,31 &, 79 \\
\hline Expresar su opinión & 1109 & 1 & 5 & 3,29 & 1,06 \\
\hline Compartir opiniones de otras personas & 1103 & 1 & 5 & 3,41 & 0,97 \\
\hline Realizar una petición a las instituciones públicas & 1100 & 1 & 5 & 3,13 & 1,08 \\
\hline Presentar una queja a las instituciones públicas & 1093 & 1 & 5 & 3,08 & 1,12 \\
\hline Formular una protesta a las instituciones públicas & 1094 & 1 & 5 & 3,11 & 1,14 \\
\hline $\begin{array}{l}\text { Apoyar iniciativas solidarias mediante la firma } \\
\text { una iniciativa solidaria }\end{array}$ & 1094 & 1 & 5 & 3,56 & 1,00 \\
\hline Inscribirse en programas de voluntariado & 1058 & 1 & 5 & 3,47 & 0,95 \\
\hline $\begin{array}{l}\text { Apoyar económicamente causas solidarias me- } \\
\text { diante donaciones }\end{array}$ & 1069 & 1 & 5 & 3,52 & 0,98 \\
\hline Competencia Digital $(\alpha=.919)$ & 1092 & 1 & 5 & 3,75 &, 75 \\
\hline $\begin{array}{l}\text { Características de la red, de los programas de } \\
\text { navegación y otras utilidades }\end{array}$ & 1113 & 1 & 5 & 3,64 & 0,93 \\
\hline $\begin{array}{l}\text { Conocimiento de los sitios de búsqueda de } \\
\text { información }\end{array}$ & 1113 & 1 & 5 & 3,80 & 0,86 \\
\hline Selección y evaluación de la información & 1108 & 1 & 5 & 3,77 & 0,84 \\
\hline Modificación de los criterios de búsqueda & 1108 & 1 & 5 & 3,77 & 0,86 \\
\hline
\end{tabular}

DOI: ri14.v15i2.1068 | ISSN: 1697-8293 | Julio - diciembre 2017 Volumen 15 N² 2 | ICONO14 


\section{ARTÍCULOS DE INVESTIGACIÓN}

\begin{tabular}{|c|c|c|c|c|c|}
\hline & $\mathrm{N}$ & Mínimo & Máximo & Media & $\begin{array}{l}\text { Desv. } \\
\text { típica }\end{array}$ \\
\hline Almacenamiento información & 1107 & 1 & 5 & 3,74 & 0,87 \\
\hline $\begin{array}{l}\text { Visibilidad y Posibilidad Acceso Datos Abier- } \\
\text { tos }(\alpha=.89)\end{array}$ & 1071 & 1 & 5 & 2,57 & ,95 \\
\hline $\begin{array}{l}\text { Las instituciones públicas se dan a conocer ade- } \\
\text { cuadamente en Internet a través de sus portales } \\
\text { y bancos abiertos }\end{array}$ & 1095 & 1 & 5 & 2,96 & 1,06 \\
\hline $\begin{array}{l}\text { Las instituciones públicas proporcionan sufi- } \\
\text { cientes datos abiertos sobre sus actividades } \\
\text { (salarios, estadísticas, etc.) }\end{array}$ & 1093 & 1 & 5 & 2,42 & 1,10 \\
\hline $\begin{array}{l}\text { Las instituciones públicas proporcionan datos } \\
\text { abiertos sobre sus actividades (salarios, estadís- } \\
\text { ticas, etc.) }\end{array}$ & 1088 & 1 & 5 & 2,39 & 1,11 \\
\hline $\begin{array}{l}\text { Las instituciones públicas ofrecen herramientas } \\
\text { y aplicaciones sencillas para analizar e inter- } \\
\text { pretar los datos abiertos que ofrecen (salarios, } \\
\text { estadísticas, etc.) }\end{array}$ & 1083 & 1 & 5 & 2,51 & 1,11 \\
\hline $\begin{array}{l}\text { Alfabetización para Uso Información Pública } \\
\text { en Internet }(\alpha=.93)\end{array}$ & 1055 & 1 & 5 & 3,42 &, 77 \\
\hline $\begin{array}{l}\text { Acceder a datos en abierto sobre las activida- } \\
\text { des y datos internos de funcionamiento de las } \\
\text { instituciones públicas }\end{array}$ & 1096 & 1 & 5 & 3,34 & 0,94 \\
\hline $\begin{array}{l}\text { Realizar una petición de información sobre las } \\
\text { actividades y datos internos de funcionamiento } \\
\text { de las instituciones públicas }\end{array}$ & 1101 & 1 & 5 & 3,28 & 0,96 \\
\hline $\begin{array}{l}\text { Localizar la información disponible en las pági- } \\
\text { nas Web de las instituciones públicas }\end{array}$ & 1108 & 1 & 5 & 3,61 & 0,88 \\
\hline $\begin{array}{l}\text { Manejar y tratar la información de las páginas } \\
\text { Web de las instituciones públicas }\end{array}$ & 1101 & 1 & 5 & 3,54 & 0,89 \\
\hline Analizar la información pública & 1104 & 1 & 5 & 3,51 & 0,89 \\
\hline $\begin{array}{l}\text { Manejar herramientas o servicios de visuali- } \\
\text { zación de información pública (Google Fusion } \\
\text { Tables, Many eyes, GeoCommons y }\end{array}$ & 1075 & 1 & 5 & 3,26 & 1,00 \\
\hline $\begin{array}{l}\text { Aplicar y compartir la información pública en } \\
\text { otros contextos y fines }\end{array}$ & 1094 & 1 & 5 & 3,41 & 0,93 \\
\hline
\end{tabular}

Tabla 3: Estadísticos descriptivos y análisis de fiabilidad de las variables independientes creadas ad hoc para la investigación 
Estudio de variables relevantes en el uso de la información publicada... | 157 ARTÍCULOS DE INVESTIGACIÓN

\begin{tabular}{|l|c|c|}
\hline \multicolumn{1}{|c|}{ Sexo } & N & Porcentaje \\
\hline \multicolumn{1}{|c|}{ Edad } & & \\
\hline Mujer (0) & 601 & 53,3 \\
\hline Hombre (1) & 526 & 46,7 \\
\hline Total & 1127 & 100 \\
\hline \multicolumn{1}{|c|}{ Estudios } & 75 & 6,7 \\
\hline Entre 16 y 18 años (1) & 196 & 17,4 \\
\hline Entre 19 y 24 años (2) & 218 & 19,3 \\
\hline Entre 25 y 34 años (3) & 233 & 20,7 \\
\hline Entre 35 y 44 años (4) & 221 & 19,6 \\
\hline Entre 45 y 54 años (5) & 184 & 16,3 \\
\hline Más de 55 años (6) & 1127 & 100 \\
\hline Total & & \\
\hline & 0 & 0 \\
\hline Sin estudios (1) & 15 & 1,3 \\
\hline Primaria (2) & 80 & 7,1 \\
\hline Secundaria obligatoria (3) & 134 & 11,9 \\
\hline Formación Profesional de Grado Medio (4) & 207 & 18,4 \\
\hline Bachillerato (5) & 158 & 14 \\
\hline Formación Profesional de Grado Superior (6) & 533 & 47,3 \\
\hline Universitarios (7) & 1127 & 100 \\
\hline Total & & \\
\hline
\end{tabular}

Tabla 4: Frecuencias de las variables sociodemográficas 